\title{
Reconsidering outcomes that matter to patients
}

\author{
Andrea Talacchi - Martin Taphoorn • \\ Gabriele Miceli
}

Published online: 7 April 2012

(C) Springer Science+Business Media, LLC. 2012

In 1977 experts gathered in Turin (Italy) at a meeting held under the auspices of the World Health Organization (WHO) to discuss the standardization of reporting the results of cancer treatment. Since then, tumor-related outcome criteria (disease progression, complete response, partial response, etc.) have become a widely applied yardstick in clinical trials. In clinical practice, surgery, chemotherapy and radiotherapy have become increasingly effective in controlling disease. Yet less is known about the patient's functioning and health-related quality of life after treatment, when improved survival may come at the cost of increased long-term "toxicity".

In neuro-oncology, the concept of patient-related outcome, comprising patient-reported outcome, objective neurological examination and performance scales, has developed at an uneven pace. Extensive study into patientreported outcome has resulted in validated questionnaires, while objective neurological examination and (non-specific) performance scales date back to decades ago. Added to these is cognitive testing, providing reliable instruments

\footnotetext{
A. Talacchi $(\square)$

Section of Neurosurgery, Department of Neurosciences,

University of Verona, P. Stefani 1, 37126 Verona, Italy

e-mail: andrea.talacchi@univr.it
}

\section{Taphoorn}

Department of Neurology, Medical Center Haaglanden,

VU University Medical Center, The Hague, The Netherlands

e-mail: m.taphoorn@mchaaglanden.nl

\section{G. Miceli}

CeRiN (Center for Neurocognitive Rehabilitation),

CIMeC (Center for Mind/Brain Sciences), University of Trento,

Via Matteo del Ben 5A, 38068 Rovereto, TN, Italy

e-mail: gabriele.miceli@unitn.it

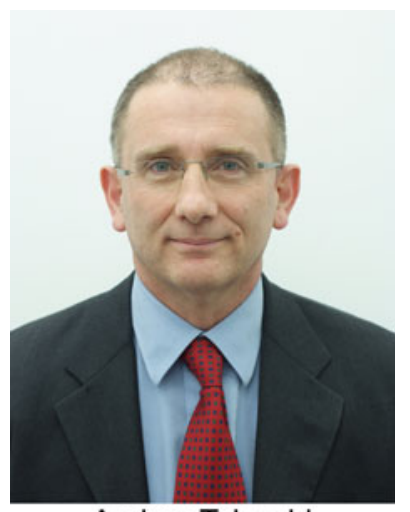

Andrea Talacchi

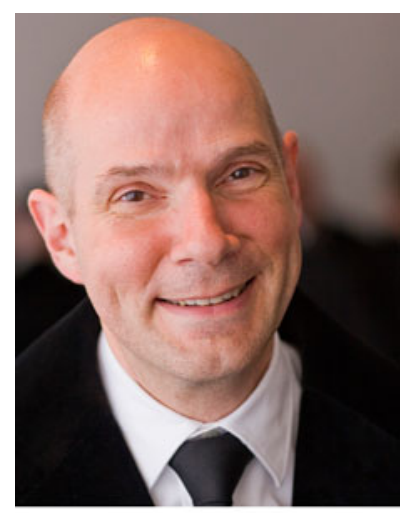

Martin Taphoorn

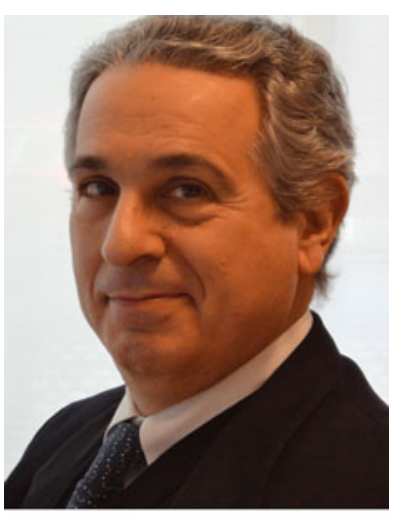

Gabriele Miceli

for generating objective psychometric data in the evaluation of specific high-order brain functions.

Following the efforts of Martin Klein and Martin Taphoorn from The Netherlands, and the pioneering work of Christina Meyers from the MD Anderson Cancer Center 
(Houston, Texas, USA), evidence-based research has underlined the need for neuropsychological instruments in cognitive assessment. To date, however, the absence of neuropsychological expertise at cancer centers has limited the extent to which cognition can be thoroughly tested. An even greater challenge is to develop methods that evaluate and assemble different sources of clinical data.

To renew attention toward improving the quality of the neurological and cognitive examination, the editors of this special issue have put together a collection of articles by researchers and clinicians who presented their work at the meeting entitled "Measuring clinical outcome in neurooncology: tools and parameters. To what extent can neuropsychology become part and parcel of clinical practice?" (January 2011, Verona, Italy) and exchanged experience on research and assessment across multidisciplinary competences.

Building on the work started more than 30 years ago, the overarching aim is to achieve an international consensus on the core outcome set in neuro-oncology, now considering the patient-related counterpart. Balanced in scope, the topics covered here will capture the interest of all those involved in the fascinating task of combating cancer while preserving brain functioning. The original and review papers presented here outline the state of the art and indicate prospects for clinical research. 